\title{
The modified Clavien classification system: a standardized platform for reporting complications in transurethral resection of the prostate
}

\author{
Charalampos Mamoulakis • Ioannis Efthimiou • \\ Savas Kazoulis • Ioannis Christoulakis • \\ Frank Sofras
}

Received: 23 February 2010/Accepted: 22 April 2010/Published online: 12 May 2010

(C) The Author(s) 2010. This article is published with open access at Springerlink.com

\begin{abstract}
Purpose The aim of the study was to evaluate the applicability of the modified Clavien classification system (CCS) in grading perioperative complications of transurethral resection of the prostate (TURP).

Methods All patients with benign prostatic hyperplasia submitted to monopolar TURP from January 2006 to February 2008 at a non-academic center were evaluated for complications occurring up to the end of the first postoperative month. All complications were classified according to the modified CCS independently by two urologists, and the final decision was based on consensus. If multiple complications per patient occurred, categorization was done in more than one grade. Results were presented as complication rates per grade.

Results Forty-four complications were recorded in 31 out of 198 patients (overall perioperative morbidity rate: $15.7 \%$ ), and their grading was generally easy, non-timeconsuming and straightforward. Most of them were classified as grade I (59.1\%) and II (29.5\%). Higher grade complications were scarce (grade III: $2.3 \%$ and grade IV: $6.8 \%$, respectively) There was one death (grade V: $2.3 \%$ ) due to acute myocardial infarction (overall mortality rate:
\end{abstract}

C. Mamoulakis · F. Sofras

Department of Urology, University of Crete, Heraklion, Greece

C. Mamoulakis $(\bowtie)$

Department of Urology (G5-250), Academic Medical Center, University of Amsterdam, Meibergdreef 9, 1105 AZ Amsterdam,

The Netherlands

e-mail: c.mamoulakis@amc.uva.nl

I. Efthimiou - S. Kazoulis · I. Christoulakis Department of Urology, General Hospital of Chania, Crete, Greece
$0.5 \%)$. Negative outcomes such as mild dysuria during this early postoperative period or retrograde ejaculation were considered sequelae and were not recorded. Nobody was complicated with severe dysuria. There was one re-operation due to residual adenoma $(0.5 \%)$.

Conclusions The modified CCS represents a straightforward and easily applicable tool that may help urologists to classify the complications of TURP in a more objective and detailed way. It may serve as a standardized platform of communication among clinicians allowing for sound comparisons.

Keywords Benign prostatic hyperplasia .

Complications · Classification - Prostate .

Transurethral resection of prostate $\cdot$ Treatment outcome

\section{Introduction}

Transurethral resection of the prostate (TURP) has been considered since decades the surgical "golden standard" for the benign prostatic hyperplasia (BPH) management. In the absence of strong evidence favouring newer technologies such as the various laser types [1] and although problems of outcome assessment might still exist [2], TURP currently remains clinically effective and cost effective [3]. Nevertheless, despite a decreasing trend over time, TURP complications still occur [4]. A large-scale prospective multicenter study showed that TURP mortality has nowadays decreased $(0.1 \%)$ but that immediate morbidity, although reduced, remains high (11.1\%) and still represents a concern [5].

Although results from several historically retrospective [6-8] and prospective [5] large multicenter cohorts focusing on TURP morbidity and mortality have been reported, 
there is still lack of consensus on how to define complications and grade their severity. This hampers sound and reproducible comparisons among centers using similar or different approaches and within a center over time. Therefore, a standardized classification offering a common platform for communication among urologists is necessary. The Clavien classification system (CCS) has been proposed to grade complications of general surgery [9]. It has recently been modified and prospectively validated in a large patient cohort submitted to elective general surgery [10]. It is increasingly becoming popular in urology but has never been used to date in common procedures such as BPH-related interventions [11]. Our aim was to evaluate the applicability of the modified CCS in reporting and grading the severity of perioperative complications in patients with BPH submitted to TURP and to discuss its benefits. To the best of our knowledge, this is the first report on perioperative TURP complications using this relatively new classification system.

\section{Materials and methods}

All patients with BPH submitted to TURP at a non-academic hospital (Department of Urology, General Hospital of Chania, Crete, Greece) from January 2006 until February 2008 were evaluated. Only new TURP cases were considered. Patients with prostate cancer at the time of the operation or incidentally diagnosed by the procedure were excluded.

All operations were performed using monopolar electrosurgical system (ERBOTOM ICC 300, ERBE Electromedizin GmbH, Tüblingen, Germany) and Karl Storz 26 F continuous flow resectoscope. The cutting and coagulation settings were 120 and 80 Watt, respectively. Sorbitol 3\% was used for bladder irrigation intra-operatively. All operations were performed under general or spinal anesthesia by consultant urologists. At operation completion, a 20 French three-way Couvelair catheter was inserted for continuous bladder irrigation with normal saline. Bladder irrigation was terminated, and catheter was removed on the first and third postoperative day, respectively, based on the department protocol, unless differently indicated. Patients were usually discharged on the following day after catheter removal.

Basic preoperative patient data were recorded, and all complications occurring during the perioperative period (up to the end of the first month after the operation) were classified prospectively according to the modified CCS (Table 1) by a junior consultant hardly involved in the operation procedures (I.E.) to diminish the well-known observation bias due to surgeon-related complication misjudgment. Subsequently, the complications recorded were classified in retrospect by a second co-author (C.M.) independently. Any disagreement was resolved by discussion, and final decision was based on consensus. In case of more than one complication in the same patient, categorization was done in more than one grade. Results were presented as complication rates per grade.

\section{Results}

Data on 198 men with BPH submitted to TURP during a two-year period were evaluated. The baseline patients' characteristics are shown in Table 2. A total of 44 complications were recorded in 31 patients during the first postoperative month (overall perioperative morbidity rate: $15.7 \%$ ). The classification according to the modified CCS is presented in Table 3. In general, the system application

Table 1 Classification of surgical complications based on the modified Clavien system [10]

\begin{tabular}{|c|c|c|}
\hline Grade & Subgrade & Definition \\
\hline I & & $\begin{array}{l}\text { Any deviation from the normal postoperative course without the need for pharmacological treatment or surgical, } \\
\text { endoscopic and radiological interventions. Allowed therapeutic regimens are drugs as antiemetics, antipyretics, } \\
\text { analgesics, diuretics, electrolytes and physiotherapy. This grade also includes wound infections opened at the bedside }\end{array}$ \\
\hline II & & $\begin{array}{l}\text { Complications requiring pharmacological treatment with drugs other than such allowed for grade I complications. Blood } \\
\text { transfusions and total parenteral nutrition are also included }\end{array}$ \\
\hline \multirow[t]{3}{*}{ III } & & Complications requiring surgical, endoscopic or radiological intervention \\
\hline & a & Intervention not under general anesthesia \\
\hline & $\mathrm{b}$ & Intervention under general anesthesia \\
\hline \multirow[t]{3}{*}{ IV } & & Life-threatening complications (including CNS complications) requiring IC/ICU management \\
\hline & a & Single organ dysfunction (including dialysis) \\
\hline & $\mathrm{b}$ & Multiorgan dysfunction \\
\hline V & & Death \\
\hline $\begin{array}{l}\text { Suffix } \\
\text { "d" }\end{array}$ & & $\begin{array}{l}\text { If the patient suffers from a complication at the time of discharge, the suffix " } \mathrm{d} \text { " (for disability) is added to the respective } \\
\text { grade of complication. This label indicates the need for a follow-up to fully evaluate the complication }\end{array}$ \\
\hline
\end{tabular}


Table 2 Baseline characteristics of patients with BPH submitted to TURP

\begin{tabular}{ll}
\hline Total number of patients & 198 \\
\hline $\begin{array}{l}\text { Number of patients with a catheter } \\
\text { preoperatively (\%) }\end{array}$ & $39(19.7)$ \\
Mean age (SD) (years) & \\
Median (IQR) prostate volume $^{\mathrm{a}}(\mathrm{mL})$ & $68.4(8.0)$ \\
Median (IQR) serum PSA level $^{\mathrm{b}}(\mathrm{ng} / \mathrm{mL})$ & $54(40-70)$ \\
Median (IQR) Qmax $^{\mathrm{b}}(\mathrm{mL} / \mathrm{s})$ & $3.0(1.7-3.9)$ \\
Median (IQR) IPSS $^{\mathrm{b}}$ & $9.0(7.0-10.0)$ \\
Median (IQR) QoL score $^{\mathrm{b}}$ & $22(20-27)$ \\
ASA score & $4(4-5)$ \\
I & $\mathrm{I}-\mathrm{III}$ \\
II & 19 \\
III & 169 \\
\hline
\end{tabular}

$B P H$ benign prostatic hyperplasia, $I Q R$ inter-quartile range, $P S A$ prostate-specific antigen, $Q o L$ quality of life, $S D$ standard deviation, TURP transurethral resection of the prostate

a Applicable in the total population

b Applicable in patients without a catheter preoperatively

was relatively simple, non-time-consuming, easy and straightforward without any special disagreement between the two independent observers.

The vast majority of the complications were classified as grade I (26 out of 44 complications; 59.1\%) and II (13 out of 44 complications; 29.5\%). Higher grade complications were scarce (grade III: 1 out of 44 complications; $2.3 \%$ and grade IV: 3 out of 44 complications; $6.8 \%$ ) There was only one fatal complication (grade V: 1 out of 44 complications; $2.3 \%$ ), i.e., death due to acute myocardial infarction (overall mortality rate: $0.5 \%$ ). Negative outcomes, such as mild dysuria during this early postoperative period not related to remarkable morbidity necessitating subsequent interventions, or retrograde ejaculation, were considered sequelae rather than complications and were not recorded. Nobody was complicated with severe dysuria. There was one case of re-operation due to residual adenoma (treatment failure; $0.5 \%$ ).

\section{Discussion}

The aim of this study was mainly to evaluate the applicability of the modified CCS in reporting and grading the severity of perioperative complications in patients with BPH submitted to TURP rather than to report on the wellknown negative procedural outcomes, which have been since long, extensively documented in historically retrospective [6-8] or prospective studies [5] on large multicenter cohorts, and summarized in narrative $[4,12]$ as well as systematic reviews [13].

The lack of a uniform way of reporting negative surgical outcomes has been recognized as an obstacle in interpreting the related literature, and the need for a standardized system to report complications following uro-oncological procedures has been acknowledged [14]. The modified CCS has been proposed as a standard tool to report complications, which should be used accordingly to increase the quality of the related urological literature [15].

Clavien et al. in 1992 proposed a structured classification using therapeutic consequences as the basis to rank surgical complications and provided a utility example for cholecystectomy [9]. The authors defined negative procedural outcomes by differentiating complications (unexpected

Table 3 Complications in the present series classified according to the modified Clavien system

\begin{tabular}{lll}
\hline Grade & Complication & Management \\
\hline I & Hematuria $(n=5) \pm$ blood clot retention $(n=5)$ & $\begin{array}{c}\text { Bedside bladder irrigation (prolonged) } \pm \text { clot } \\
\text { evacuation } \pm \text { catheter traction }\end{array}$ \\
& Catheter malfunction due to either clot or chip block $(n=3)$ & $\begin{array}{l}\text { Bedside catheter change } \\
\text { Bedside recatheterization }\end{array}$ \\
& Transient elevation of serum creatinine $(n=2)$ & Watchful regulation of fluid balance \\
& Low urinary tract infection $(n=7)$ & Antibiotics \\
II & Intraoperative hemorrhage/Hematuria $(n=8)$ & Transfusion \\
& Urinary tract infection with signs of bacteremia $(n=2)$ & Antibiotics \\
& Supraventricular tachycardia $(n=2)$ & Antiarrythmic agents \\
IIIb & Pulmonary embolism $(n=1)$ & Anticoagulants \\
& Extraperitoneal fluid collection due to subtrigonal catheter & Endoscopic catheter reposition and surgical drainage \\
IVa & location and malfunction $(n=1)$ & under anesthesia \\
IVb & Acute myocardial infarction $(n=2)$ & Admission to intensive care unit \\
$\mathrm{V}$ & Transurethral resection syndrome $(n=1)$ & Admission to intensive care unit \\
\hline
\end{tabular}


events not intrinsic to the operation) from sequelae ("aftereffects" inherent to the procedure) and failures (procedural aim not fulfilled). However, some events may contain elements of both sequelae and complications, such as severe postoperative pain, and should be considered complications.

Procedural complications were classified into four grades as follows: grade I for any alterations from the ideal postoperative course, which are non-life-threatening, nonresidual disability resulting; grade II for any potentially life-threatening, non-residual disability resulting complications (further subdivided into IIa and IIb according to the requirement for invasive procedures); grade III for any residual disability resulting complications; and grade IV for any lethal complications.

In 2004, Dindo et al. modified the original criteria to increase the accuracy and applicability of the CCS, [10] keeping the definitions for complications, sequelae and failures as well as the cornerstone concept of using the therapeutic consequences for ranking the complications. Modifications mainly focused on the manner of reporting life-threatening and permanently disabling conditions. In brief, grades I, IIa and IIb correspond to grades I, II and III (further subdivided into grades IIIa and IIIb depending on the need for general anesthesia) in the new version, respectively, while life-threatening complications have been recognized to be of higher grade (grade IV). Hospitalization length as a criterion to rank grade II complications has been eliminated, and disability has been highlighted by the suffix "d", not representing any more a distinct grade (grade III in the original version). The new classification has been tested in a cohort of 6,336 patients submitted to elective general surgery, and its reproducibility has been evaluated with an international survey of 144 surgeons of different training levels from 10 centers. The authors concluded that the modified CCS is reliable and may represent a compelling tool for quality assessment of surgery worldwide.

The power of such an approach relies on the principle of grading based on the therapy used to treat complications, which is usually well documented in physician and nursing reports. Although postoperative problems, especially the minor ones, are often poorly reported, the use of therapeutic consequences as the basis to rank complications allows for detecting most of them and prevents down-rating of major negative outcomes, a feature particularly important in retrospective analyses. Consequently, such a system offers potential advantages allowing for increased uniformity in reporting results, for longitudinal comparisons of the results within a center, for comparisons of the results among centers and for conduction of adequate metaanalyses. Nevertheless, the management of a given surgical complication may vary among physicians, centers or countries mostly due to the lack of accepted paradigm for the "best practice" or availability of medical resources, which may be considered an inherent limitation. Furthermore, it may still allow for some subjectivity in individual surgeons' recording their complications or interpret the way that particular complications should be graded.

The urological community has only recently started to adopt the system for grading complications of oncologic procedures such as radical prostatectomy, cystectomy and renal surgery [16-25]. However, its use in non-oncologic procedures remains still limited [26-30], and it has never been used to date in BPH-related interventions [11]. To the best of our knowledge, this is the first report on the use of this system for classifying complications of TURP in the international literature.

A total of 44 complications in 31 out of 198 patients were encountered up to the first postoperative month. In general, we did not experience any difficulties in assigning a grade to these complications and there was no special disagreement between the two independent observers. Therefore, the application of the modified CCS was considered relatively simple, non-time-consuming, easy and straightforward.

The vast majority of the patients experienced either no complication (167 out of $198 ; 84.4 \%$ ) or complications ranked as grade I-II (27 out of 198; 13.6\%), which were merely related to persisting bleeding, catheter placement or replacement and urinary tract infections (UTI). Only 3 out of 198 patients (1.5\%) experienced life-threatening complications necessitating intensive care unit admission (grade IV). In general, the results on specific complication rates are in accordance with large series [4, 5], which underlines that the present series may be considered representative and adequate for the assessment of a complication reporting system.

Regarding bleeding (intraoperative hemorrhage or postoperative hematuria), the most "severe" therapeutic consequence was the need for transfusion, a clear grade II complication. Blood transfusion was considered necessary due to significant postoperative hemoglobin drop in relation to the general and cardiac conditions of the patients in 8 out of 198 cases $(4.0 \%)$. Apart from the need for transfusion, the therapeutic consequences of bleeding were minor including simple bedside interventions only; therefore, these complications were clearly graded as I by both observers. A total of 10 patients presented with hematuria, which resolved spontaneously after a relatively prolonged bladder irrigation alone ( 5 patients; $2.5 \%$ ) or resulted in bladder tamponade ( 5 patients; $2.5 \%$ ) during the first 24 postoperative hours, necessitating repetitive bedside manual blood clot evacuation via the catheter with or without application of catheter traction. No patient required reintervention for bleeding control. 
Complications necessitating bedside bladder catheterization alone (catheter replacement due to malfunction of the existent catheter in 3 patients; $1.5 \%$ or acute urine retention after catheter removal in 4 patients; $2 \%$ ) were also clearly classified as grade I by both observers, based on the fact that the original CCS considers "retention treated medically or by catheterization alone" as grade I complication, which corresponds to the same grade in the new version as well. Acute urine retentions after catheter removal resolved spontaneously after being treated conservatively with a1-blockers and bladder drainage for 2-3 days, without need for further intervention.

A total of 9 patients (4.5\%) experienced UTI necessitating antibiotic treatment. However, 7 of them experienced symptoms suggestive of low UTI and the rest presented signs of bacteremia (fever $>38.5^{\circ} \mathrm{C}$ ). UTI necessitating antibiotics were initially graded collectively as grade II (Table 1). However, after a more careful consideration of both the original and the modified CCS, the final consensus was that these complications should be differentiated. Consequently, low UTI were considered grade I based on the original CCS, which corresponds to grade I also in the modified version. The cases of urosepsis were classified according to the original CCS as grade IIa, which corresponds to grade II in the modified version.

One patient presented with a large retroperitoneal fluid collection due to subtrigonal catheter misplacement at the end of the operation. This rare complication was treated with catheter reposition in the theater under endoscopic guidance and fluid drainage in the same session with a preperitoneal drain placement under anesthesia (grade IIIb). Among the three patients experiencing life-threatening complications that necessitated intensive care unit admission (grade IV), one (0.5\%) presented with TUR syndrome with symptoms and signs from the central nervous and the cardiovascular system, while the rest $(1 \%)$ experienced acute myocardial infarction and one of them died (grade V). The assignment of these complications into the respective higher grades was straightforward by both observers.

In the present series, patients were followed up systematically up to the first postoperative month. Therefore, TURP complications, which typically appear in the longer term, were not graded. Nevertheless, we feel that such complications can also be easily classified based on the same principles. For example, a case of erectile dysfunction that necessitates medical treatment with phosphodiesterase 5 inhibitors should be considered grade II complication. A case of bladder neck stenosis or urethral stricture necessitating bladder neck incision or urethrotomy under anesthesia, respectively, should be considered grade IIIb complication.

\section{Conclusion}

The modified CCS provides a validated method that has already been successfully adopted by several urological centers for grading complications of mainly major oncologic procedures. Based on our experience, the aforementioned system is non-time-consuming, easily applicable tool for grading perioperative TURP complications. Despite the inherent limitations, it seems that it may well serve as a straightforward, standardized platform allowing for sound comparisons, either longitudinally within centers to facilitate audit or among centers using similar or different technologies such as monopolar vs. bipolar TURP.

Acknowledgments Dr Charalampos Mamoulakis was partially supported by the Alexander S. Onassis Public Benefit Foundation and the European Urological Scholarship Programme for a clinical fellowship at the AMC, Department of Urology, Amsterdam, The Netherlands.

Conflict of interest statement The authors declare that they have no conflict of interest.

Open Access This article is distributed under the terms of the Creative Commons Attribution Noncommercial License which permits any noncommercial use, distribution, and reproduction in any medium, provided the original author(s) and source are credited.

\section{References}

1. Herrmann TR, Bach T, Imkamp F et al (2010) Thulium laser enucleation of the prostate (ThuLEP): transurethral anatomical prostatectomy with laser support. Introduction of a novel technique for the treatment of benign prostatic obstruction. World $\mathbf{J}$ Urol 28:45-51

2. Mishriki SF, Grimsley SJ, Nabi G, Cohen NP (2010) Partners agree that the treatment of LUTS reduces patients' bother and improves their quality of life: prospective 12 years follow-up study. World J Urol 28:123-132

3. Lourenco T, Armstrong N, N'Dow J et al (2008) Systematic review and economic modelling of effectiveness and cost utility of surgical treatments for men with benign prostatic enlargement. Health Technol Assess 12:1-516

4. Rassweiler J, Teber D, Kuntz R, Hofmann R (2006) Complications of transurethral resection of the prostate (TURP)-incidence, management, and prevention. Eur Urol 50:969-980

5. Reich O, Gratzke C, Bachmann A et al (2008) Morbidity, mortality and early outcome of transurethral resection of the prostate: a prospective multicenter evaluation of 10,654 patients. J Urol 180:246-249

6. Holtgrewe HL, Valk WL (1962) Factors influencing the mortality and morbidity of transurethral prostatectomy: a study of 2, 015 cases. J Urol 87:450-459

7. Melchior J, Valk WL, Foret JD, Mebust WK (1974) Transurethral prostatectomy: computerized analysis of 2, 223 consecutive cases. J Urol 112:634-642

8. Mebust WK, Holtgrewe HL, Cockett AT, Peters PC (1989) Transurethral prostatectomy: immediate and postoperative complications. A cooperative study of 13 participating institutions evaluating 3, 885 patients. J Urol 141:243-247 
9. Clavien PA, Sanabria JR, Strasberg SM (1992) Proposed classification of complications of surgery with examples of utility in cholecystectomy. Surgery 111:518-526

10. Dindo D, Demartines N, Clavien PA (2004) Classification of surgical complications: a new proposal with evaluation in a cohort of a 6336 patients and results of a survey. Ann Surg 240:205-213

11. Morgan M, Smith N, Thomas K, Murphy D (2009) Is Clavien the new standard for reporting urological complications? BJU Int 4:434-439

12. Mamoulakis C, Trompetter M, de la Rosette J (2009) Bipolar transurethral resection of the prostate: the "golden standard" reclaims its leading position. Curr Opin Urol 19:26-32

13. Mamoulakis C, Ubbink DT, de la Rosette JJ (2009) Bipolar versus monopolar transurethral resection of the prostate: a systematic review and meta-analysis of randomized controlled trials. Eur Urol 56:798-809

14. Donat SM (2007) Standards for surgical complication reporting in urologic oncology: time for a change. Urology 69:221-225

15. Graefen M (2010) The modified Clavien system: a plea for a standardized reporting system for surgical complications. Eur Urol 57:387-389

16. Novara G, Ficarra V, D'Elia C et al (2010) Prospective evaluation with standardized criteria for postoperative complications after robotic-assisted laparoscopic radical prostatectomy. Eur Urol 57:363-370

17. Carlsson S, Nilsson AE, Schumacher MC et al. (2009) Surgeryrelated complications in 1253 robot-assisted and 485 open retropubic radical prostatectomies at the Karolinska University Hospital, Sweden. Urology [Epub ahead of print]

18. Constantinides CA, Tyritzis SI, Skolarikos A et al (2009) Shortand long-term complications of open radical prostatectomy according to the Clavien classification system. BJU Int 103:336340

19. Stolzenburg JU, Rabenalt R, Do M et al (2008) Endoscopic extraperitoneal radical prostatectomy: the University of Leipzig experience of 2000 cases. J Endourol 22:2319-2325
20. Pruthi RS, Nielsen ME, Nix J et al (2010) Robotic Radical Cystectomy for bladder cancer: surgical and pathological outcomes in 100 consecutive cases. J Urol 183:510-515

21. Ng CK, Kauffman EC, Lee MM et al (2010) A comparison of postoperative complications in open versus robotic cystectomy. Eur Urol 57:274-282

22. Kauffman EC, Ng CK, Lee MM et al (2009) Critical analysis of complications after robotic-assisted radical cystectomy with identification of preoperative and operative risk factors. BJU Int 105:520-527

23. Novara G, De Marco V, Aragona M et al (2009) Complications and mortality after radical cystectomy for bladder transitional cell cancer. J Urol 182:914-921

24. Hautmann RE, de Petriconi R, Volkmer BG (2009) Neobladder formation after pelvic irradiation. World J Urol 27:57-62

25. Laguna MP, Beemster P, Kumar P et al (2009) Perioperative morbidity of laparoscopic cryoablation of small renal masses with ultrathin probes: a European multicentre experience. Eur Urol 56:355-361

26. Wezel F, Mamoulakis C, Rioja J et al (2009) Two contemporary series of percutaneous tract dilation for percutaneous nephrolithotomy. J Endourol 23:1655-1661

27. de la Rosette JJ, Zuazu JR, Tsakiris P et al (2008) Prognostic factors and percutaneous nephrolithotomy morbidity: a multivariate analysis of a contemporary series using the Clavien classification. J Urol 180:2489-2493

28. Tefekli A, Ali Karadag M, Tepeler K et al (2008) Classification of percutaneous nephrolithotomy complications using the modified Clavien grading system: looking for a standard. Eur Urol 53:184-190

29. Hemal AK, Mishra S (2010) Retroperitoneoscopic nephrectomy for pyonephrotic nonfunctioning kidney. Urology 75:585-588

30. Rassweiler JJ, Teber D, Frede T et al (2008) Complications of laparoscopic pyeloplasty. World J Urol 26:539-547 